\title{
On Bisectors for Convex Distance Functions
}

\author{
Chan He, Horst Martini, Senlin Wu \\ College of Management, Harbin University of Science and Technology, \\ 150080 Harbin, China, chan_he@hotmail.com \\ Faculty of Mathematics, Chemnitz University of Technology, \\ 09107 Chemnitz, Germany, horst.martini@mathematik.tu-chemnitz.de \\ Department of Applied Mathematics, Harbin University of Science and Technology, \\ 150080 Harbin, China, wusenlin@outlook.com
}

Received February 26, 2013

\begin{abstract}
It is well known that the construction of Voronoi diagrams is based on the notion of bisector of two given points. Already in normed linear spaces, bisectors have a complicated structure and can, for many classes of norms, only be described with the help of topological methods. Even more general, we present results on bisectors for convex distance functions (gauges). Let $C$, with the origin $o$ from its interior, be the compact, convex set inducing a convex distance function (gauge) in the plane, and let $B(-x, x)$ be the bisector of $-x$ and $x$, i.e., the set of points $z$ whose distance (measured with the convex distance function induced by $C$ ) to $-x$ equals that to $x$. For example, we prove the following characterization of the Euclidean norm within the family of all convex distance functions: if the set $L$ of points $x$ in the boundary $\partial C$ of $C$ that create $B(-x, x)$ as a straight line has non-empty interior with respect to $\partial C$, then $C$ is an ellipse centered at the origin. For the subcase of normed planes we give an easier approach, extending the result also to higher dimensions.
\end{abstract}

Key words: Birkhoff orthogonality, bisector, characterization of ellipse, convex distance function, Euclidean norm, gauge, isosceles orthogonality, Roberts orthogonality, Voronoi diagram.

AMS Subject Class. (2010): 46B20, 52A10.

\section{INTRODUCTION}

It is well known that for the construction of Voronoi diagrams the notion of bisector of two given points is fundamental. To construct bisectors in Euclidean spaces is an elementary task, but to investigate them in general normed spaces can be, from the geometric and topological viewpoint, very difficult, since bisectors can even be full-dimensional sets. For results on bisectors and Voronoi diagrams in normed linear spaces we refer to the survey [14]. Here we want to prove some new theorems on bisectors for convex distance functions, which are more general than respective statements for norms. More precisely, for a planar convex body $C$ taken as unit ball of a 


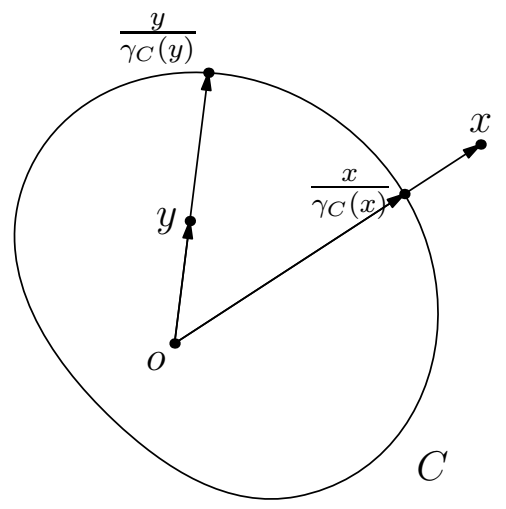

Figure 1: A planar convex body and the corresponding gauge.

convex distance function (gauge) we show the following: If the set $L$ of all points $x$ in the boundary of $C$ that create their bisectors $B(-x, x)$ (see the definition below) as straight lines has nonempty interior with respect to that boundary of $C$, then $C$ is an ellipse centered at the origin. An easier approach to that characterization of Euclidean geometry, which is also available for higher dimensions, is applied to the subcase when $C$ is centrally symmetric with respect to the origin, i.e., when the gauge $\gamma_{C}$ (see the definition below) is a norm.

Let $C \subseteq \mathbb{R}^{2}$ be a convex body (i.e., a compact, convex set with non-empty interior $\operatorname{int} C$ ) satisfying $o \in \operatorname{int} C$, and $\partial C$ be the boundary of $C$. The gauge (or Minkowski functional) $\gamma_{C}$, defined by

$$
\begin{aligned}
\gamma_{C}: \mathbb{R}^{2} & \rightarrow \mathbb{R} \\
x & \rightarrow \inf \{\lambda>0: x \in \lambda C\},
\end{aligned}
$$

has the following properties (see Figure 1 and, for instance, [7, p. 128-130]):

1. $\gamma_{C}(x) \geq 0 \quad \forall x \in \mathbb{R}^{2}$,

2. $\gamma_{C}(x)=0$ if and only if $x$ is the origin $o$,

3. $\gamma_{C}(\lambda x)=\lambda \gamma_{C}(x) \quad \forall x \in \mathbb{R}^{2}, \lambda \geq 0$,

4. $\gamma_{C}(x+y) \leq \gamma_{C}(x)+\gamma_{C}(y)$.

The convex distance function $d_{C}(\cdot, \cdot)$ induced by $C$ is then defined via $\gamma_{C}(x)$ by:

$$
d_{C}(y, x):=\gamma_{C}(x-y) .
$$

Clearly, $d_{C}(\cdot, \cdot)$ satisfies the following properties. 
1. $d_{C}(y, x)=d_{C}(y+z, x+z) \quad \forall x, y, z \in \mathbb{R}^{2}$,

2. $d_{C}(\alpha y, \alpha x)=\alpha d_{C}(y, x) \quad \forall \alpha \geq 0$.

Notice that $d_{C}(\cdot, \cdot)$ is not necessarily symmetric, i.e., in general $d_{C}(p, q) \neq$ $d_{C}(q, p)$. The distance function $d_{C}(\cdot, \cdot)$ is symmetric if and only if $C$ is symmetric with respect to the origin $o$, in which case $\gamma_{C}$ is a norm.

By $[p, q]$ we denote the segment (possibly degenerate) between two points $p, q \in X$, by $[p, q\rangle$ the ray with starting point $p$ passing through $q(p \neq q)$, and by $\langle p, q\rangle$ the line passing through $p$ and $q(p \neq q)$.

For each point $x \in \partial C$, we denote by $x^{-}$the point in which the ray $[x, o\rangle$ intersects $\partial C$. Also, each point $x \in \partial C$ is associated with a number $\mu_{x}>0$ such that $x^{-}=-\mu_{x} x$. Let $u$ and $v$ be two linearly independent points in $\partial C$. Then we call the set

$$
\operatorname{arc}(u, v):=\{\lambda u+\mu v: \lambda, \mu \geq 0\} \cap \partial C
$$

the (minor) arc of $\partial C$ connecting $u$ and $v$. Let $L$ be the set of points $x$ in $\partial C$ such that $B(-x, x)$ is a straight line, where $B(-x, x)$ is the bisector of $-x$ and $x$, which is defined by

$$
B(-x, x):=\left\{z \in \mathbb{R}^{2}: d_{C}(-x, z)=d_{C}(x, z)\right\} .
$$

More generally, the bisector $B(p, q)$ of two distinct points $p$ and $q$ is defined by

$$
B(p, q):=\left\{z \in \mathbb{R}^{2}: d_{C}(p, z)=d_{C}(q, z)\right\} .
$$

Due to various applications (such as Voronoi diagrams, see [5], [12], and [13]), bisectors are deeply studied in Computational Geometry. But also in Minkowski Geometry they play an increasing role; see [8], [9], [14], and [16]. Of course, since the convex distance function is not necessarily symmetric, there are other ways to define bisectors. For example, we can put the set

$$
B^{\prime}(-x, x):=\left\{z \in \mathbb{R}^{2}: d_{C}(z,-x)=d_{C}(z, x)\right\} .
$$

to be the bisector of the points $-x$ and $x$. One can easily verify that $B(-x, x)=$ $-B^{\prime}(-x, x)$.

The set $L$ introduced above can be empty, even if $C$ is symmetric with respect to the origin. Such an example can be found in [10, Example 2.1]. The aim of the paper is to show that, if the interior of $L$ with respect to $\partial C$ is not empty, then $C$ is an ellipse centered at the origin. 
In Section 2 we deal with the general planar case, namely when $C$ is not necessarily symmetric with respect to the origin. Extending our investigations also to higher dimensions, we study in Section 3 the special case when $C$ is symmetric with respect to the origin $o$. Benefiting from recent results in Functional Analysis, our approach in Section 3 is much easier. It is also working for the case when $C$ is the unit ball of an infinite dimensional real normed linear space, in which case $C$ is a bounded closed (but not compact) convex set with non-empty interior, which is symmetric with respect to the origin.

\section{The General CASE}

The results in this section hold for $\mathbb{R}^{2}$. We note that if $x \in L$, then $x^{-}=-\mu_{x} x \in L$, which can be easily seen from the following equality:

$$
B\left(-\mu_{x} x, \mu_{x} x\right)=\mu_{x} B(-x, x)
$$

The following lemma describes the relation between the structure of a bisector and a property of $\partial C$.

Lemma 2.1. (cf. [13, Lemma 2.1.1.1, Corollary 2.1.1.2]) Let $x$ be a point distinct from $o$. Then $B(-x, x)$ is homeomorphic to a line if and only if there is no non-trivial segment contained in $\partial C$ and parallel to $\langle-x, x\rangle$.

By Lemma 2.1, if $B(-x, x)$ is homeomorphic to a line, then the two supporting lines of $C$ which are parallel to the line $\langle-x, x\rangle$ intersect $C$, in each case, in precisely one point.

Lemma 2.2. If $x \in L$, then there exist precisely two points $n_{x}$ and $s_{x}$ in $\partial C$ such that the lines $n_{x}+\langle-x, x\rangle$ and $s_{x}+\langle-x, x\rangle$ are the two supporting lines of $C$ which are parallel to $\langle-x, x\rangle$. Moreover, $o \in\left[n_{x}, s_{x}\right]$.

Proof. First, since $B(-x, x)$ is a straigth line, there exist precisely two points $n_{x}$ and $s_{x}$ in $\partial C$ such that the lines $n_{x}+\langle-x, x\rangle$ and $s_{x}+\langle-x, x\rangle$ are two supporting lines of $C$. For each point $z \in B(-x, x)$ we have the inequality

$$
\alpha(z):=d_{C}(-x, z)=d_{C}(o, z+x) \leq d_{C}(o, z)+1 .
$$


Also, we have

$$
\begin{aligned}
d_{C}(o, z) & =d_{C}\left(o, \frac{1}{2}(z+x)+\frac{1}{2}(z-x)\right) \\
& \leq d_{C}\left(o, \frac{1}{2}(z+x)\right)+d_{C}\left(o, \frac{1}{2}(z-x)\right) \\
& =\frac{1}{2} d_{C}(-x, z)+\frac{1}{2} d_{C}(x, z)=\frac{1}{2} \alpha(z)+\frac{1}{2} \alpha(z)=\alpha(z) .
\end{aligned}
$$

From (1) and (2) it follows that

$$
1 \geq d_{C}\left(o, \frac{1}{\alpha(z)} z\right) \geq \frac{d_{C}(o, z)}{d_{C}(o, z)+1} .
$$

Thus, when $d_{C}(o, z)$ tends to infinity, $d_{C}\left(o, \frac{1}{\alpha(z)} z\right)$ tends to 1 . In the meantime, since $\alpha(z)=d_{C}(-x, z)=d_{C}(x, z)$,

$$
d_{C}\left(o, \frac{1}{\alpha(z)}(z+x)\right)=d_{C}\left(o, \frac{1}{\alpha(z)}(z-x)\right)=1 .
$$

Thus $\frac{1}{\alpha(z)} z$ is the midpoint of the chord $\left[\frac{1}{\alpha(z)}(z+x), \frac{1}{\alpha(z)}(z-x)\right]$ of $C$ which is parallel to $\langle-x, x\rangle$.

Denote by $H_{x}^{+}$and $H_{x}^{-}$the two open halfplanes bounded by $\langle-x, x\rangle$ and containing $n_{x}$ and $s_{x}$, respectively. If $\left\{z_{n}\right\}_{n=1}^{\infty}$ is a sequence contained in $H_{x}^{+} \cap B(-x, x)$ such that $d_{C}\left(o, z_{n}\right)$ tends to infinity, then $\frac{z_{n}}{\alpha\left(z_{n}\right)}$ tends to $n_{x}$ since $\left[\frac{z_{n}+x}{\alpha\left(z_{n}\right)}, \frac{z_{n}-x}{\alpha\left(z_{n}\right)}\right]$ is a chord of $C$ parallel to $\langle-x, x\rangle$, whose midpoint $\frac{z_{n}}{\alpha\left(z_{n}\right)}$ has distance to the origin tending to 1 . Similarly, if $\left\{z_{n}\right\}_{n=1}^{\infty}$ is a sequence contained in $H_{x}^{-} \cap B(-x, x)$ such that $d_{C}\left(o, z_{n}\right)$ tends to infinity, then $\frac{z_{n}}{\alpha\left(z_{n}\right)}$ tends to $s_{x}$.

Next we show that $o \in\left[n_{x}, s_{x}\right]$. Suppose that this is not true. Then we can suitably choose a pair of lines $l$ and $l^{\prime}$ from the following four lines: the two supporting lines of $C$ which are parallel to $\left\langle n_{x}, s_{x}\right\rangle$ and the two lines parallel to $\left\langle n_{x}, s_{x}\right\rangle$ and passing through $x$ and $-x$, respectively. "Suitably" here means that the other two lines lie between them. Without loss of generality, we may assume that $l$ and $o$ are separated by the line $\left\langle n_{x}, s_{x}\right\rangle$. We denote by $X^{+}$the halfplane bounded by $l$ which does not contain $C$. From foregoing discussions, there exist two points $z_{1}$ and $z_{2}$ in $B(-x, x)$ such that $z_{1}$ lies in the halfplane bounded by $\langle-x, x\rangle$ containing $n_{x}$, and $z_{2}$ lies in the other halfplane containing $s_{x}$. These two points $z_{1}$ and $z_{2}$ can be chosen so that $\frac{z_{1}}{d_{C}\left(o, z_{1}\right)}$ and $\frac{z_{2}}{d_{C}\left(o, z_{2}\right)}$ are "sufficiently close to" $n_{x}$ and $s_{x}$, respectively. Here "sufficiently close to" means that the rays $\left[o, z_{1}\right\rangle$ and $\left[o, z_{2}\right\rangle$ both intersect $l$. 
Also, the numbers $d_{C}\left(o, z_{1}\right)$ and $d_{C}\left(o, z_{2}\right)$ can be chosen large enough such that $z_{1}$ and $z_{2}$ are both in $X^{+}$. Then the line $\left\langle z_{1}, z_{2}\right\rangle$, which is precisely $B(-x, x)$, intersects the line $\langle-x, x\rangle$ but does not touch the relative interior of the segment $[-x, x]$. This is impossible and leads to a contradiction.

By Lemma 2.2, we can associate each $x \in L$ with two points $n_{x}$ and $s_{x}$ such that

1. $n_{x}+\langle-x, x\rangle$ and $s_{x}+\langle-x, x\rangle$ are the two supporting lines of $C$ which are parallel to $\langle-x, x\rangle$;

2. $n_{x}$ is contained in the part of $\partial C$ that connects $x$ with $x^{-}$counterclockwise.

LEMMA 2.3. If $x \in L$, then

$$
B(-x, x)=\frac{1-\mu_{x}}{1+\mu_{x}} x+\left\langle n_{x}, s_{x}\right\rangle .
$$

Proof. First we show that $B(-x, x)$ is parallel to the line $\left\langle n_{x}, s_{x}\right\rangle$. Suppose to the contrary that this is not true. Let $\left\{z_{n}\right\} \subset B(-x, x)$ be a sequence, which is contained in the halfplane bounded by $\langle-x, x\rangle$ and containing $n_{x}$, such that $\lim _{n \rightarrow \infty} d_{C}\left(o, z_{n}\right)=\infty$. We claim that

$$
\lim _{n \rightarrow \infty} \frac{z_{n}}{d_{C}\left(o, z_{n}\right)} \neq n_{x}
$$

which is a contradiction to the fact that $\frac{z_{n}}{\alpha\left(z_{n}\right)}$ tends to $n_{x}$ (cf. the proof of Lemma 2.2). Otherwise,

$$
\lim _{n \rightarrow \infty} \frac{z_{n}-p}{d_{C}\left(o, z_{n}\right)}=n_{x}
$$

where $p$ is the point of intersection of the lines $\left\langle n_{x}, s_{x}\right\rangle$ and $B(-x, x)$. By Lemma 2.2, $o \in\left[n_{x}, s_{x}\right]$. It follows that the lines $\left\langle n_{x}, s_{x}\right\rangle$ and $B(-x, x)$ coincide, which is a contradiction.

Next we show that $\frac{1-\mu_{x}}{1+\mu_{x}} x \in B(-x, x)$, which follows directly from the equations

$$
\begin{aligned}
d_{C}\left(x, \frac{1-\mu_{x}}{1+\mu_{x}} x\right) & =d_{C}\left(o,-\frac{2 \mu_{x}}{1+\mu_{x}} x\right)=\frac{2}{1+\mu_{x}} d_{C}\left(o,-\mu_{x} x\right) \\
& =\frac{2}{1+\mu_{x}}=\frac{2}{1+\mu_{x}} d_{C}(o, x) \\
& =d_{C}\left(o, \frac{2}{1+\mu_{x}} x\right)=d_{C}\left(-x, \frac{1-\mu_{x}}{1+\mu_{x}} x\right) .
\end{aligned}
$$




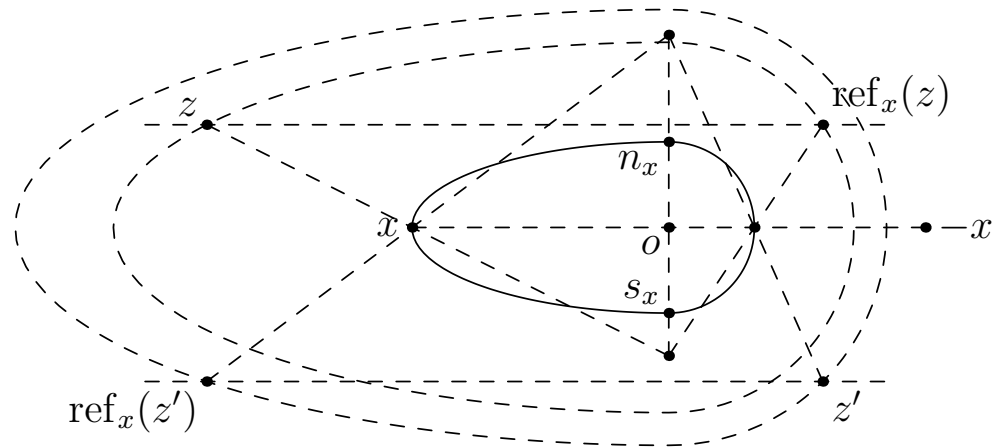

Figure 2: The definition of $\operatorname{ref}_{x}(z)$.

Lemma 2.4. If $x \in L$, then, for each number $\beta \in \mathbb{R}$,

$$
d_{C}\left(o,-\frac{2 \mu_{x}}{1+\mu_{x}} x+\beta n_{x}\right)=d_{C}\left(o, \frac{2}{1+\mu_{x}} x+\beta n_{x}\right) .
$$

Proof. From Lemma 2.3 and the relation $o \in\left[n_{x}, s_{x}\right]$ it follows that, for each number $\beta \in \mathbb{R}$,

$$
\frac{1-\mu_{x}}{1+\mu_{x}} x+\beta n_{x} \in B(-x, x) .
$$

Thus

$$
d_{C}\left(o, \frac{1-\mu_{x}}{1+\mu_{x}} x+\beta n_{x}-x\right)=d_{C}\left(o, \frac{1-\mu_{x}}{1+\mu_{x}} x+\beta n_{x}+x\right),
$$

from which (3) follows.

Let $x$ be a point in $L$. For any point $z=\alpha x+\beta n_{x}=-\frac{1}{\mu_{x}} \alpha x^{-}+\beta n_{x} \in \mathbb{R}^{2}$, set (see Figure 2)

$$
\operatorname{ref}_{x}(z)= \begin{cases}-\frac{1}{\mu_{x}} \alpha x+\beta n_{x}, & \alpha<0 \\ \alpha x^{-}+\beta n_{x}, & \alpha \geq 0\end{cases}
$$

Lemma 2.5. Let $x$ be a point in $L$. Then, for each point $z \in \mathbb{R}^{2}$, we have

1. $\operatorname{ref}_{x}\left(\operatorname{ref}_{x}(z)\right)=z$,

2. $d_{C}(o, z)=d_{C}\left(o, \operatorname{ref}_{x}(z)\right)$. 
Proof. Assume that $z=\alpha x+\beta n_{x}$.

1)

$$
\begin{aligned}
\operatorname{ref}_{x}\left(\operatorname{ref}_{x}(z)\right) & = \begin{cases}\operatorname{ref}_{x}\left(-\frac{1}{\mu_{x}} \alpha x+\beta n_{x}\right), & \alpha<0, \\
\operatorname{ref}_{x}\left(-\mu_{x} \alpha x+\beta n_{x}\right), & \alpha \geq 0,\end{cases} \\
& =\alpha x+\beta n_{x} .
\end{aligned}
$$

2) If $\alpha<0$, then, by Lemma 2.4,

$$
\begin{aligned}
d_{C}\left(o, \alpha x+\beta n_{x}\right) & =-\alpha \cdot \frac{1+\mu_{x}}{2 \mu_{x}} d_{C}\left(o,-\frac{2 \mu_{x}}{1+\mu_{x}} x-\frac{\beta}{\alpha} \cdot \frac{2 \mu_{x}}{1+\mu_{x}} n_{x}\right) \\
& =-\alpha \cdot \frac{1+\mu_{x}}{2 \mu_{x}} d_{C}\left(o, \frac{2}{1+\mu_{x}} x-\frac{\beta}{\alpha} \cdot \frac{2 \mu_{x}}{1+\mu_{x}} n_{x}\right) \\
& =d_{C}\left(o,-\frac{\alpha}{\mu_{x}} x+\beta n_{x}\right) \\
& =d_{C}\left(o, \operatorname{ref}_{x}(z)\right) .
\end{aligned}
$$

If $\alpha>0$, then

$$
\begin{aligned}
d_{C}\left(o, \alpha x+\beta n_{x}\right) & =\alpha \cdot \frac{1+\mu_{x}}{2} d_{C}\left(o, \frac{2}{1+\mu_{x}} x+\frac{\beta}{\alpha} \cdot \frac{2}{1+\mu_{x}} n_{x}\right) \\
& =\alpha \cdot \frac{1+\mu_{x}}{2} d_{C}\left(o,-\frac{2 \mu_{x}}{1+\mu_{x}} x+\frac{\beta}{\alpha} \cdot \frac{2}{1+\mu_{x}} n_{x}\right) \\
& =d_{C}\left(o,-\mu_{x} \alpha x+\beta n_{x}\right) \\
& =d_{C}\left(o, \operatorname{ref}_{x}(z)\right) .
\end{aligned}
$$

If $\alpha=0$, then

$$
d_{C}(o, z)=d_{C}\left(o, \beta n_{x}\right)=d_{C}\left(o, \operatorname{ref}_{x}(z)\right) .
$$

Lemma 2.6. If $x$ and $y$ are two linearly independent points in $\partial C$ and $\operatorname{arc}(x, y) \subset L$, then there exists a number $\gamma_{0}$ such that

$$
\operatorname{arc}\left(n_{x}, n_{y}\right)=\gamma_{0} \operatorname{arc}\left(s_{x}, s_{y}\right) .
$$

Proof. We note that, since $C$ is a convex curve, for each smooth point $w \in \operatorname{arc}\left(n_{x}, n_{y}\right)$ there exists a unique point $z \in \operatorname{arc}(x, y)$ such that $w=n_{z}$. Let $f_{1}(\theta)(\cos (\theta), \sin (\theta))\left(\theta \in\left[\theta_{1}, \theta_{2}\right]\right)$ and $f_{2}(\theta+\pi)(\cos (\theta+\pi), \sin (\theta+\pi))$ 
$\left(\theta \in\left[\theta_{1}, \theta_{2}\right]\right)$ be the polar equations of $\operatorname{arc}\left(n_{x}, n_{y}\right)$ and $\operatorname{arc}\left(s_{x}, s_{y}\right)$, respectively. Then

$$
\frac{f_{1}^{\prime}(\theta)}{f_{1}(\theta)}=\frac{f_{2}^{\prime}(\theta+\pi)}{f_{2}(\theta+\pi)}
$$

holds for all $\theta \in\left[\theta_{1}, \theta_{2}\right]$ with a countable set of exceptions. By integration, (4) yields

$$
\frac{f_{1}(\theta)}{f_{1}\left(\theta_{1}\right)}=\frac{f_{2}(\theta+\pi)}{f_{2}\left(\theta_{1}+\pi\right)}, \forall \theta \in\left[\theta_{1}, \theta_{2}\right] .
$$

Thus

$$
\frac{f_{1}(\theta)}{f_{2}(\theta+\pi)}=\frac{f_{1}\left(\theta_{1}\right)}{f_{2}\left(\theta_{1}+\pi\right)}, \forall \theta \in\left[\theta_{1}, \theta_{2}\right]
$$

Hence there exists a number $\gamma_{0}$ such that $\operatorname{arc}\left(n_{x}, n_{y}\right)=\gamma_{0} \operatorname{arc}\left(s_{x}, s_{y}\right)$.

To continue our discussion we need the forthcoming Lemma 2.8, which proves a seemingly obvious fact. In its proof we use the following lemma from [13].

Lemma 2.7. (cf. Lemma 2.1.2.13 in [13]) For three points $a_{1}, a_{2}, a_{3}$, we have $B\left(a_{1}, a_{2}, a_{3}\right):=B\left(a_{1}, a_{2}\right) \cap B\left(a_{1}, a_{3}\right)=\emptyset$ if and only if either $a_{3}$ is contained in the interior of the set $F G_{12}$ or $a_{3}$ lies on one of the boundary line segments of $F G_{12}$ and the tangent to $C$, where this line segments stems from, does not contain a boundary line segment of $\partial C$.

Lemma 2.8. Let $C \subset \mathbb{R}^{2}$ be a convex body containing the origin o in its interior, and $d_{C}(\cdot, \cdot)$ be the convex distance function induced by $C$. Then, for any two pairs of distinct points $a_{1}$ and $a_{2}, a_{3}$ and $a_{4}$ such that the segment $\left[a_{1}, a_{2}\right]$ is not parallel to $\left[a_{3}, a_{4}\right], B\left(a_{1}, a_{2}\right)$ and $B\left(a_{3}, a_{4}\right)$ cannot be two parallel straight lines.

Proof. Suppose to the contrary that $B\left(a_{1}, a_{2}\right)$ and $B\left(a_{3}, a_{4}\right)$ are two parallel straight lines. First, observe that for any $\alpha>0$ and any vectors $p, q, z$, the following equalities hold 


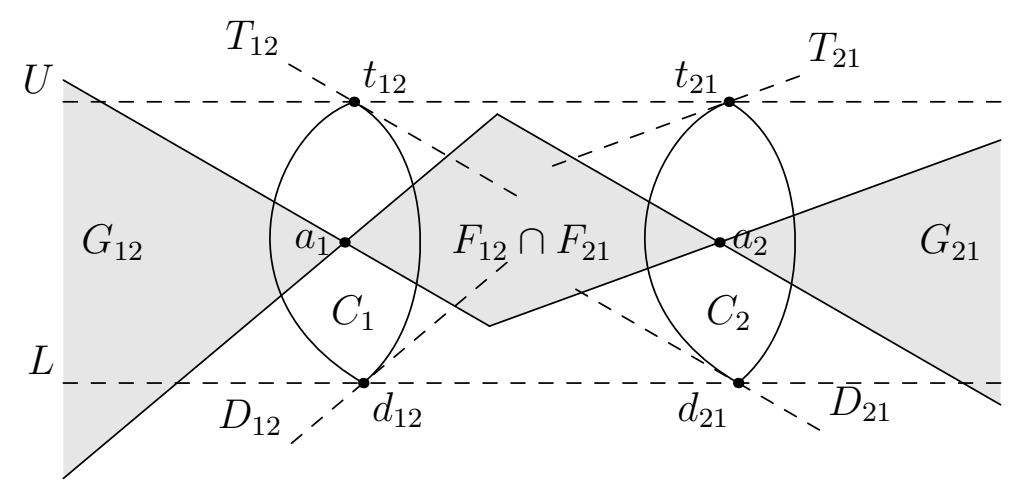

Figure 3: $F G_{12}$ is the shaded region.

$$
\begin{aligned}
B(\alpha p+z, \alpha q+z) & =\left\{w: d_{C}(\alpha p+z, w)=d_{C}(\alpha q+z, w)\right\} \\
& =\left\{w: d_{C}\left(p+\frac{1}{\alpha} z, \frac{1}{\alpha} w\right)=d_{C}\left(q+\frac{1}{\alpha} z, \frac{1}{\alpha} w\right)\right\} \\
& =\left\{w: d_{C}\left(p, \frac{1}{\alpha}(w-z)\right)=d_{C}\left(q, \frac{1}{\alpha}(w-z)\right)\right\} \\
& =\left\{\alpha v+z: d_{C}(p, v)=d_{C}(q, v)\right\} \\
& =\alpha\left\{v: d_{C}(p, v)=d_{C}(q, v)\right\}+z \\
& =\alpha B(p, q)+z
\end{aligned}
$$

Thus we may assume, without loss of generality, that $a_{4}=a_{1}$.

Next we take some terminology from [13], to apply Lemma 2.7. Without loss of generality we assume that the line $\left\langle a_{1}, a_{2}\right\rangle$ is horizontal. Let $t_{12}$ and $d_{12}$ be the top and bottom point of $C_{1}:=C+a_{1}$, respectively; $t_{21}$ and $d_{21}$ be the top and bottom point of $C_{2}:=C+a_{2}$, respectively; and let $U$ and $L$ be the upper and lower common supporting lines of $C_{1}$ and $C_{2}$, respectively.

Let $T_{21}$ be the steepest tangent to $C_{2}$ at $t_{21}$, and let $T_{12}$ be the least steep tangent to $C_{1}$ at $t_{12}$. Correspondingly, let $D_{12}$ be the steepest tangent to $C_{1}$ at $d_{12}$, and let $D_{21}$ be the least steep tangent to $C_{2}$ at $d_{21}$.

We consider the four cones with apex $a_{1}$ defined by the lines through $a_{1}$ parallel to $T_{12}$ and $D_{12}$, respectively. Let $F_{12}$ denote the cone bounded by the line parallel to $D_{12}$ from above and by the line parallel to $T_{12}$ from below, and let $G_{12}$ be the opposite cone. Analogously we have $F_{21}$ and $G_{21}$ with apex $a_{2}$. For brevity let $F G_{12}$ denote the set $G_{12} \cup\left(F_{12} \cap F_{21}\right) \cup G_{21}$, see Figure 3 . 
On the line $\left\langle a_{1}, a_{3}\right\rangle$ there is precisely one point $a$ such that the bisectors $B\left(a_{1}, a_{2}\right)$ and $B\left(a_{1}, a\right)$ intersect. Since the lines $\left\langle a_{1}, a_{2}\right\rangle$ and $\left\langle a_{1}, a\right\rangle$ do not coincide, there exists a point $a_{3}^{\prime}$ distinct from $a$ on the line $\left\langle a_{1}, a\right\rangle \backslash F G_{12}$. Then $B\left(a_{1}, a_{2}, a_{3}^{\prime}\right):=B\left(a_{1}, a_{2}\right) \cap B\left(a_{1}, a_{3}^{\prime}\right)=\emptyset$, since $B\left(a_{1}, a_{3}^{\prime}\right)$ is a straight line parallel to $B\left(a_{1}, a_{3}\right)$. This is a contradiction to Lemma 2.7 .

Lemma 2.9. If $x$ lies in the closure of the interior of $L$ with respect to $\partial C$, then $\mu_{x}=1$.

Proof. First suppose that $x$ is in the interior of $L$ in $\partial C$. Then there exist two linearly independent points $u, v \in L$ such that

$$
x \in \operatorname{arc}(u, v) \subset L .
$$

If $n_{u}=n_{x}$, then, by Lemma 2.2, $s_{u}=s_{x}$. By Lemma 2.3, $B(-u, u)$ is parallel to $B(-x, x)$, which is in contradiction to Lemma 2.8. Thus $n_{u} \neq n_{x}$. Similarly, $n_{v} \neq n_{x}$.

Without loss of generality, these two points $u$ and $v$ are chosen such that

$$
n_{v}=\operatorname{ref}_{x}\left(n_{u}\right) \text { and } n_{u}=\alpha x+\beta n_{x},
$$

where $\alpha, \beta>0$. Then from the definition of $\operatorname{ref}_{x}$ it follows that

$$
n_{v}=-\mu_{x} \alpha x+\beta n_{x} .
$$

Lemma 2.6 shows that there exists a number $\gamma_{0}>0$ such that

$$
\operatorname{arc}\left(s_{u}, s_{v}\right)=-\gamma_{0} \operatorname{arc}\left(n_{u}, n_{v}\right) .
$$

Thus

$$
s_{u}=-\gamma_{0} n_{u}=-\gamma_{0} \alpha x-\gamma_{0} \beta n_{x}
$$

and

$$
s_{v}=-\gamma_{0} n_{v}=\gamma_{0} \mu_{x} \alpha x-\gamma_{0} \beta n_{x} .
$$

From (5) it follows that the line $\left\langle s_{u}, s_{v}\right\rangle$ is parallel to the line $\langle-x, x\rangle$. By Lemma 2.1, $\left\{s_{u}, s_{v}\right\}=\left\langle s_{u}, s_{v}\right\rangle \cap \partial C$. Since $s_{u}, s_{v} \in \partial C$, by the definition of ref $_{x}$ and Lemma 2.5 we have

$$
\begin{aligned}
\gamma_{0} \mu_{x} \alpha x-\gamma_{0} \beta n_{x} & =s_{v}=\operatorname{ref}_{x}\left(s_{u}\right)=\operatorname{ref}_{x}\left(-\gamma_{0} \alpha x-\gamma_{0} \beta n_{x}\right) \\
& =\frac{1}{\mu_{x}} \gamma_{0} \alpha x-\gamma_{0} \beta n_{x}
\end{aligned}
$$

which implies that $\mu_{x}=\frac{1}{\mu_{x}}$. Thus $\mu_{x}=1$.

One can easily verify that $\mu_{x}$ is continuous with respect to $x$. Thus, for each point $y$ in the closure of the interior of $L$ with respect to $\partial C, \mu_{y}=1$. 


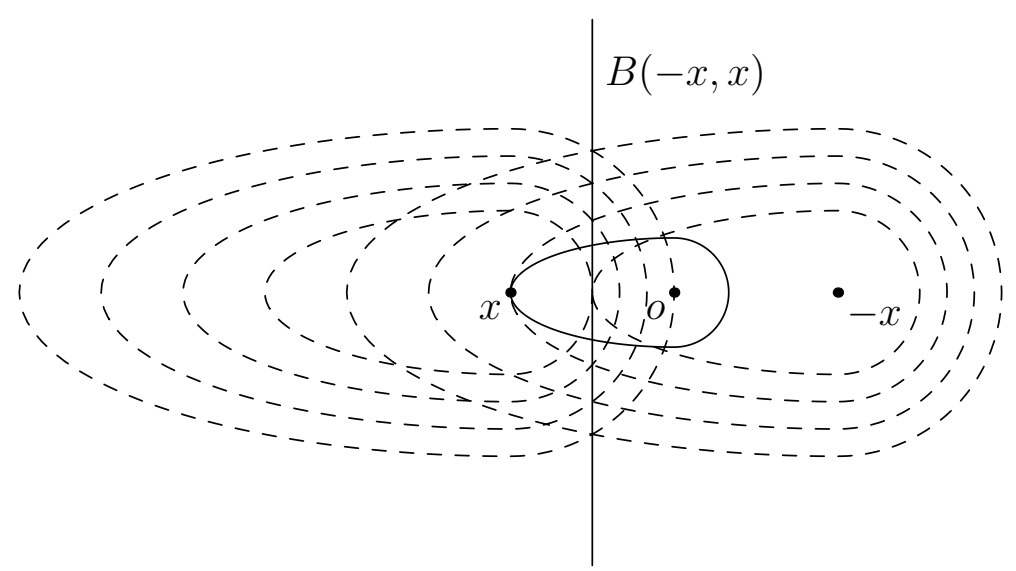

Figure 4: A bisector $B(-x, x)$ which is a line not passing through the origin.

Corollary 2.10. If $x \in \partial C$ lies in the interior of $L$ in $\partial C$, then

1. $B(-x, x)$ contains the origin,

2. $\operatorname{ref}_{x}(z)=-\alpha x+\beta n_{x}$ holds for any point $z=\alpha x+\beta n_{x}$, and

3. ref $_{x}$ is linear.

Proof. 1) Since, by Lemma 2.3,

$$
\frac{1-\mu_{x}}{1+\mu_{x}} x \in B(-x, x)
$$

it follows from Lemma 2.9 that $o \in B(-x, x)$.

2) This is an easy consequence of the definition of $\operatorname{ref}_{x}$ and the fact that $\mu_{x}=1$.

3) Let $z_{1}=\alpha_{1} x+\beta_{1} n_{x}$ and $z_{2}=\alpha_{2} x+\beta_{2} n_{x}$ be two arbitrary points in $\mathbb{R}^{2}$, and $\lambda, \gamma$ be two arbitrary real numbers. Then

$$
\begin{aligned}
\operatorname{ref}_{x}\left(\lambda z_{1}+\gamma z_{2}\right) & =\operatorname{ref}_{x}\left(\left(\lambda \alpha_{1}+\gamma \alpha_{2}\right) x+\left(\lambda \beta_{1}+\gamma \beta_{2}\right) n_{x}\right) \\
& =-\left(\lambda \alpha_{1}+\gamma \alpha_{2}\right) x+\left(\lambda \beta_{1}+\gamma \beta_{2}\right) n_{x} \\
& =\lambda \operatorname{ref}_{x}\left(\alpha_{1} x+\beta_{1} n_{x}\right)+\gamma \operatorname{ref}_{x}\left(\alpha_{2} x+\beta_{2} n_{x}\right) \\
& =\lambda \operatorname{ref}_{x}\left(z_{1}\right)+\gamma \operatorname{ref}_{x}\left(z_{2}\right) .
\end{aligned}
$$

This implies that ref $_{x}$ is linear. 
Remark 2.11. Without further assumptions, $B(-x, x)$ being a straight line does not imply that $B(-x, x)$ is a straight line containing the origin. Here is an example: Let $C$ be a convex body in $\mathbb{R}^{2}$, the polar coordinate $\rho=\rho(\theta)$ of whose boundary $\partial C$ satisfies the following conditions (cf. Figure 4):

1. For each $\theta \in[0, \pi / 2]$ and each $\theta \in[3 \pi / 2,2 \pi), \rho(\theta)=1$.

2. For each $\theta \in(\pi / 2, \pi), \rho(\theta)=\sin (\arctan (3 \tan (\pi-\theta))) / \sin (\pi-\theta)$. And $\rho(\pi)=3$.

3. For each $\theta \in(\pi, 3 \pi / 2), \rho(\theta)=\sin (\arctan (3 \tan (\theta-\pi))) / \sin (\theta-\pi)$.

Let $x=(-3,0)$ and $o^{\prime}=(-1.5,0)$. We claim that $B(-x, x)$ is the straight line $o^{\prime}+\langle(0,1),(0,-1)\rangle$.

In fact, for any $z \in o^{\prime}+[o,(0,1)\rangle$, the polar angles $\theta$ and $\theta^{\prime}$ of vectors $z-x$ and $z-(-x)$, respectively, satisfy $\theta \in[0, \pi / 2)$ and

$$
\tan \theta^{\prime}=-\frac{\left\|z-o^{\prime}\right\|_{E}}{\left\|-x-o^{\prime}\right\|_{E}}=-\frac{\left\|z-o^{\prime}\right\|_{E}}{\left\|x-o^{\prime}\right\|_{E}} \cdot \frac{\left\|x-o^{\prime}\right\|_{E}}{\left\|-x-o^{\prime}\right\|_{E}}=-\frac{1}{3} \tan \theta,
$$

where $\|p-q\|_{E}$ stands for the Euclidean distance between two points $p$ and $q$. Then

$$
\theta^{\prime}=\pi+\arctan \left(-\frac{1}{3} \tan \theta\right)=\pi-\arctan \left(\frac{1}{3} \tan \theta\right) \in\left(\frac{\pi}{2}, \pi\right] .
$$

For any point $z \in\left(o^{\prime}+[o,(0,1)\rangle\right) \backslash\left\{o^{\prime}\right\}$, the polar angle $\theta^{\prime}$ of $z-(-x)$ is in $(\pi / 2, \pi)$. In this case we have the equation

$$
\rho\left(\theta^{\prime}\right)=\frac{\sin \theta}{\sin \left(\pi-\theta^{\prime}\right)} .
$$

It follows that

$$
\begin{aligned}
\frac{d_{C}(x, z)}{d_{C}(-x, z)} & =\frac{\|z-x\|_{E}}{\rho(\theta)} \cdot \frac{\rho\left(\theta^{\prime}\right)}{\|z-(-x)\|_{E}} \\
& =\frac{\|z-x\|_{E} \sin \theta}{\|z-(-x)\|_{E} \sin \left(\pi-\theta^{\prime}\right)} \cdot \frac{\sin \left(\pi-\theta^{\prime}\right)}{\sin \theta} \cdot \frac{\rho\left(\theta^{\prime}\right)}{\rho(\theta)} \\
& =\frac{\left\|z-o^{\prime}\right\|_{E}}{\left\|z-o^{\prime}\right\|_{E}} \cdot \frac{\sin \left(\pi-\theta^{\prime}\right)}{\sin \theta} \cdot \frac{\rho\left(\theta^{\prime}\right)}{\rho(\theta)}=1 .
\end{aligned}
$$

For the case of $o^{\prime}$, the polar angle of $o^{\prime}-(-x)$ is $\pi$. Thus

$$
\frac{d_{C}\left(x, o^{\prime}\right)}{d_{C}\left(-x, o^{\prime}\right)}=\frac{\left\|o^{\prime}-x\right\|_{E}}{\rho(0)} \cdot \frac{\rho(\pi)}{\left\|o^{\prime}-(-x)\right\|_{E}}=\frac{1.5}{1} \cdot \frac{3}{4.5}=1,
$$


which implies that $o^{\prime} \in B(-x, x)$. Hence

$$
o^{\prime}+[o,(0,1)\rangle \subset B(-x, x)
$$

In a similar way, the inclusion

$$
o^{\prime}+[o,(0,-1)\rangle \subset B(-x, x)
$$

can be proved. Hence

$$
o^{\prime}+\langle(0,1),(0,-1)\rangle \subset B(-x, x) .
$$

Notice that, by Lemma 2.1, $B(-x, x)$ is homeomorphic to a straight line. Thus

$$
B(-x, x)=o^{\prime}+\langle(0,1),(0,-1)\rangle .
$$

Also, it can be seen from Figure 4 that, even if $B(-x, x)$ is a straight line, the set of midpoints of chords of $C$, which are parallel to $\langle-x, x\rangle$, is not necessarily contained in a line.

Lemma 2.12. If $x \in \partial C$ lies in the interior of $L$ with respect to $\partial C$ and $y$ is a point in $L$, then $\operatorname{ref}_{x}(y) \in L$.

Proof. From Lemma 2.5 and Corollary 2.10 it follows that

$$
\begin{aligned}
B(-y, y) & =\left\{z: d_{C}(-y, z)=d_{C}(y, z)\right\} \\
& =\left\{z: d_{C}(o, z+y)=d_{C}(o, z-y)\right\} \\
& =\left\{z: d_{C}\left(o, \operatorname{ref}_{x}(z+y)\right)=d_{C}\left(o, \operatorname{ref}_{x}(z-y)\right)\right\} \\
& =\left\{z: d_{C}\left(o, \operatorname{ref}_{x}(z)+\operatorname{ref}_{x}(y)\right)=d_{C}\left(o, \operatorname{ref}_{x}(z)-\operatorname{ref}_{x}(y)\right)\right\} \\
& =\left\{z: d_{C}\left(-\operatorname{ref}_{x}(y), \operatorname{ref}_{x}(z)\right)=d_{C}\left(\operatorname{ref}_{x}(y), \operatorname{ref}_{x}(z)\right)\right\} .
\end{aligned}
$$

Thus

$$
\operatorname{ref}_{x}(B(-y, y))=B\left(-\operatorname{ref}_{x}(y), \operatorname{ref}_{x}(y)\right) .
$$

Since $\operatorname{ref}_{x}$ is linear, $B\left(-\operatorname{ref}_{x}(y), \operatorname{ref}_{x}(y)\right)$ is also a straight line. Therefore $\operatorname{ref}_{x}(y) \in L$.

THEOREM 2.13. If the interior of $L$ with respect to $\partial C$ is not empty, then $C$ is an ellipse centered at the origin. 
Proof. If $L=\partial C$ then, by Corollary 2.10, $o \in B(-x, x)$ holds for every $x \in \partial C$. This implies that $C$ is symmetric with respect to the origin $o$. This, together with the assumption that $B(-x, x)$ is a straight line for each point $x \in \partial C$, implies that $C$ is an ellipse centered at the origin (cf. [4, pp. 26-28]).

Thus we only need to show that $L=\partial C$. If this is not true, then there exists a point $z \in \partial C \backslash\{L\}$. It follows that $z^{-}$is also not in $L$. Let $x \in L$ be a relative interior point of $L$ with respect to $\partial C$, and $L_{x}$ be the maximal connected component of $L$ containing $x$. Then $L_{x}$ is contained in one of the open halfplanes bounded by the line $\left\langle z^{-}, z\right\rangle$. Hence there exist two points $u$ and $v$ in $\partial C$ such that the closure of $L_{x}$ is $\operatorname{arc}(u, v)$. Now we know that the interior of $-\operatorname{arc}(u, v)$ with respect to $\partial C$ is contained in $L$. Let $u^{\prime}$ be a point in the relative interior of $\operatorname{arc}\left(u, \frac{u+x}{d_{C}(o, u+x)}\right)$ with respect to $\partial C$. By the definition of $\operatorname{ref}_{u^{\prime}}$, the line $\left\langle\operatorname{ref}_{u^{\prime}}(-x),-x\right\rangle$ is parallel to the line $\left\langle-u^{\prime}, u^{\prime}\right\rangle$. Thus $\operatorname{ref}_{u^{\prime}}(-x) \notin \operatorname{arc}(u, v)$. Moreover,

$$
\operatorname{ref}_{u^{\prime}}\left(\operatorname{arc}\left(-u^{\prime},-x\right)\right)=\left\{\operatorname{ref}_{u^{\prime}}(y): y \in \operatorname{arc}\left(-u^{\prime},-x\right)\right\}=\operatorname{arc}\left(\operatorname{ref}_{u^{\prime}}(-x), u^{\prime}\right) .
$$

From Lemma 2.12 it follows that $\operatorname{arc}\left(u^{\prime}, \operatorname{ref}_{u^{\prime}}(-x)\right) \subset L$. This contradicts the fact that $L_{x}$ is the maximal connected component of $L$ containing $x$.

\section{A Characterization OF INNER PRODUCT SPACES}

In this section, $C$ is the unit ball $B_{X}$ of a real normed linear space $X$ with norm $\|\cdot\|$, whose unit sphere is the boundary of $B_{X}$ and denoted by $S_{X}$. In this situation, $C$ is a closed bounded convex body (which is not necessarily compact) with $o$ as interior point and center of symmetry. A normed linear space is called a Banach space if it is complete. A Banach space $X$ is a Hilbert space if the norm $\|\cdot\|$ is compatible with an inner product on the linear space $X$. Although we study the general case when $X$ is not necessarily finite dimensional, our method belongs to the geometry of finite dimensional Banach spaces (or Minkowski spaces, cf. [15], [14], and the monograph [18]).

Some notions of generalized orthogonality types in normed linear spaces are needed for the discussion in the sequel. Let $x$ and $y$ be in $X$. We say that $x$ is isosceles orthogonal to $y$ if the equality

$$
\|x+y\|=\|x-y\|
$$

holds, and for this situation we write $x \perp_{I} y ; x$ is said to be Roberts orthogonal to $y$ if the equality

$$
\|x+\alpha y\|=\|x-\alpha y\|
$$


holds for any number $\alpha \in \mathbb{R}$, and we write $x \perp_{R} y$ for this case. The implication

$$
\forall x, y \in X, x \perp_{R} y \Rightarrow x \perp_{I} y
$$

is trivial while its reverse, namely

$$
\forall x, y \in X, x \perp_{I} y \Rightarrow x \perp_{R} y,
$$

forces $X$ to be an inner product space (cf. [10], [4], [2], or [3]). In other words, isosceles orthogonality is in general not homogeneous. We notice that

$$
B(-x, x)=\{z \in X:\|z-x\|=\|z+x\|\}=\left\{z \in X: z \perp_{I} x\right\} .
$$

We also need the notion of Birkhoff orthogonality. $x$ is said to be Birkhoff orthogonal to $y$ if the inequality

$$
\|x+\lambda y\| \geq\|x\|
$$

holds for any number $\lambda \in \mathbb{R}$, and in this case we write $x \perp_{B} y$.

Geometrically, $x \perp_{B} y$ means that, when $\|x\|\|y\| \neq 0$, there exists a line which contains $x$, is parallel to the line passing through $-y$ and $y$, and supports the disc of radius $\|x\|$ centered at $o$ in the two-dimensional subspace spanned by $x$ and $y$ (cf. Figure 5). One can also verify the implication

$$
\forall x, y \in X, x \perp_{R} y \Rightarrow x \perp_{B} y .
$$

For more information about the structure of bisectors, also in view of relations to properties of generalized orthogonality types (especially isosceles and Birkhoff orthogonality), we refer to [14], [16], [8], and [9].

Still we denote by $L$ the set of points $x$ in $S_{X}$ such that $B(-x, x)$ is a hyperplane. Notice that, since $B_{X}$ is symmetric with respect to the origin, $B(-x, x)$ always contains the origin $o$.

A subset $R$ of a topological space $T$ is said to be rare in $T$ if the interior of the closure of $R$ in $T$ is empty.

An operator on a real Banach space of the form $s_{e, e^{*}}: x \rightarrow x-2 e^{*}(x) e$ is called a reflection, where $e \in X$ and $e^{*} \in X^{*}$ (the dual space of $X$ ) satisfy $e^{*}(e)=1$. For a point $e \in S_{X}$ there exists at most one $e^{*} \in S_{X^{*}}$ such that $s_{e, e^{*}}$ is an isometric reflection. When there exists such an $e^{*}$ we say that $e$ is a vector of isometric reflection and that $e^{*}$ is the isometric reflection functional associated to $e$. We refer to [17], [6], and [1] for more about isometric reflections and isometric reflection vectors. 


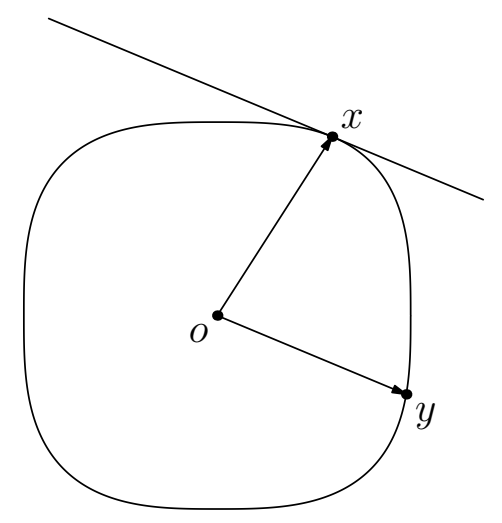

Figure 5: Birkhoff orthogonality.

Lemma 3.1. Let $X$ be a real Banach space, $e \in S_{X}, e^{*} \in S_{X^{*}}$, and $s_{e, e^{*}}$ be a reflection. Then $s_{e, e^{*}}$ is an isometric reflection if and only if

$$
e \perp_{R} H:=\left\{x: x \in X, e^{*}(x)=0\right\} .
$$

Proof. First we recall that $x \perp_{R} y \Rightarrow x \perp_{B} y$ holds for any $x, y \in X$.

Now suppose that $s_{e, e^{*}}$ is an isometric reflection. Then for any point $z \in H$ and any real number $\alpha$ we have that

$$
s_{e, e^{*}}(e+\alpha z)=\alpha z-e .
$$

Since $s_{e, e^{*}}$ is a linear isometry, we have

$$
\|e+\alpha z\|=\|e-\alpha z\|,
$$

which implies that $e \perp_{R} z$. Thus $e \perp_{R} H$.

Now suppose that $e \perp_{R} H$ holds. Clearly, $H$ is a hyperplane of $X$. Then for any point $y \in X$ there exists a point $z \in H$ such that $y=e^{*}(y) e+z$. Then

$$
\begin{aligned}
\left\|s_{e, e^{*}}(y)\right\| & =\left\|e^{*}(y) e+z-2 e^{*}\left(e^{*}(y) e+z\right) e\right\| \\
& =\left\|z-e^{*}(y) e\right\|=\left\|e^{*}(y) e+z\right\|=\|y\|,
\end{aligned}
$$

which implies that $s_{e, e^{*}}$ is an isometry.

Lemma 3.2. Let $e$ be a point in $S_{X}$. If $B(-e, e)$ is a hyperplane, then $e$ is an isometric reflection vector. 
Proof. Let $H$ be the hyperplane $B(-e, e)$. First we show that $e \perp_{R} H$. For each point $z \in H=B(-e, e)$, it is clear that $e \perp_{I} z$. Since $H$ is a hyperplane passing through the origin $o$ (this is because $o \in B(-e, e)$ ), we have

$$
\{\lambda z: \lambda \in \mathbb{R}\} \subset H .
$$

Thus $e \perp_{I} \lambda z$ holds for each number $\lambda \in \mathbb{R}$, which implies that $e \perp_{R} z$. Since $z$ is arbitrary in $H, e \perp_{R} H$.

Note that the relation $e \perp_{R} H$ implies that $e \perp_{B} H$. Then there exists a point $e^{*}$ in $S_{X^{*}}$ such that $e^{*}(e)=1$ and (cf. [11, Theorem 2.1])

$$
H=\left\{x: x \in X, e^{*}(x)=0\right\} .
$$

Then $s_{e, e^{*}}$ is a reflection and

$$
e \perp_{R}\left\{x: x \in X, e^{*}(x)=0\right\} .
$$

By Lemma 3.1, $s_{e, e^{*}}$ is an isometric reflection and $e$ is an isometric reflection vector.

Remark 3.3. The reverse of Lemma 3.2 is not true. Take, for example, the normed (or Minkowski) plane $X=\left(\mathbb{R}^{2},\|\cdot\|_{\infty}\right)$. Let $e=(1,0)$. Then $e$ is an isometric reflection vector. To see this, we put $e^{*}=(1,0)$. Then $e^{*} \in S_{X^{*}}$, $e^{*}(e)=1$, and

$$
e \perp_{R}\{\lambda(0,1): \lambda \in \mathbb{R}\}=\left\{x: x \in X, e^{*}(x)=0\right\} .
$$

However, $B(-e, e)$ is not a hyperplane, but even a set with nonempty interior (cf. Figure 6).

Lemma 3.4. (cf. [6]) A Banach space $X$ is a Hilbert space if and only if the set of all isometric reflection vectors in $X$ is not rare in $S_{X}$.

The following is the result that we announced.

Theorem 3.5. A Banach space $X$ is a Hilbert space (or, $B_{X}$ is an ellipsoid) if and only if the set $L$ is not rare in $S_{X}$.

Proof. The necessity is obvious, so we only need to show the sufficiency. By Lemma 3.2, the set $L$ is a subset of the set of isometric reflection vectors in $X$. Since $L$ is not rare in $S_{X}$, the set of isometric reflection vectors is also not rare in $S_{X}$. Then it follows from Lemma 3.4 that $X$ is a Hilbert space. 


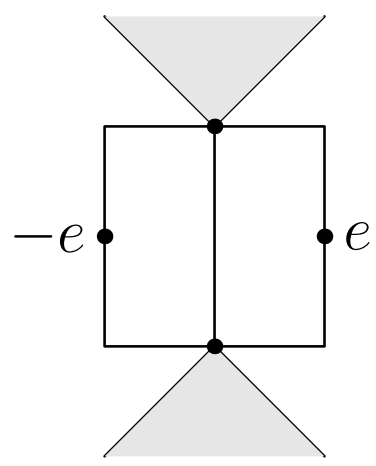

Figure 6: $B(-e, e)$ is not a hyperplane.

In the following we extend Theorem 2.13 to higher dimensional cases. Now we assume that $C \subset \mathbb{R}^{n}(n \geq 2)$ is a convex body containing the origin $o$ in its interior. Let $L$ be the set of points $x \in \partial C$ such that $B(-x, x)$ is a hyperplane. Then we have the following theorem.

TheOREM 3.6. If the interior of the set $L$ with respect to $\partial C$ is not empty, then $C$ is an ellipsoid centered at the origin.

Proof. By Theorem 2.13 we only need to deal with the subcase when $n>2$, and by Theorem 3.5 it suffices to show that $C$ is centered at the origin. Indeed, if this is true, then $\|\cdot\|=\gamma_{C}$ is a norm on $\mathbb{R}^{n}$ and the interior of the set $L$ with respect to $S_{X}=\partial C$ is not empty. By Theorem $3.5, X=\left(\mathbb{R}^{n},\|\cdot\|\right)$ is a Hilbert space and $B_{X}=C$ is an ellipsoid.

Let $x$ be an interior point of $L$ with respect to $\partial C$, and $z$ be an arbitrary point in $\partial C$ such that $x$ and $z$ are linearly independent. We denote by $X_{x, z}$ the two-dimensional subspace of $\mathbb{R}^{n}$ spanned by $x$ and $z$, and by $C_{0}$ the intersection of $C$ and $X_{x, z}$. Then it is clear that $x$ is an interior point of $L \cap \partial C_{0}$ with respect to $\partial C_{0}$. By Theorem 2.13, $C_{0}$ is an ellipse centered at the origin. This implies that $\{-x,-z\} \subset \partial C_{0} \subset \partial C$. Since $z$ is arbitrary, it follows that $C$ is centered at the origin.

\section{ACKNOWLEDGEMENTS}

The first and third named authors are grateful for financial support from the following grants: National Nature Science Foundation of China (NSFC) (grant number: 11001068), a foundation from the Ministry of Education of Heilongjiang Province (grant number 1251H013), China Postdoctoral Science Foundation (grant number 2012M520097), and the 
Science Research Foundation for the Returned Overseas Chinese Scholars, State Education Ministry.

\section{REFERENCES}

[1] A. Aizpuru, F.J. García-Pacheco, F. Rambla, Isometric reflection vectors in Banach spaces, J. Math. Anal. Appl. 299 (1) (2004), 40-48.

[2] J. Alonso, C. Benítez, Orthogonality in normed linear spaces: a survey, I, Main properties, Extracta Math. 3 (1) (1988), 1-15.

[3] J. Alonso, H. Martini, S. Wu, On Birkhoff orthogonality and isosceles orthogonality in normed linear spaces, Aequationes Math. 83 (1-2) (2012), $153-189$.

[4] D. Amir, "Characterizations of Inner Product Spaces", Operator Theory: Advances and Applications, 20, Birkhäuser Verlag, Basel, 1986.

[5] F. Aurenhammer, Voronoi diagrams-a survey of a fundamental geometric data structure, ACM Comput. Surv. 23 (3) (1991), 345-405.

[6] J.B. Guerrero, A.R. Palacios, Isometric reflections on Banach spaces after a paper of A. Skorik and M. Zaidenberg: "On isometric reflections in Banach spaces" [Mat. Fiz. Anal. Geom. 4(1-2) (1997), 212-247], Rocky Mountain J. Math. 30 (1) (2000), 63-83.

[7] J.-B. Hiriart-Urruty, C. Lemaréchal, "Fundamentals of Convex Analysis", Grundlehren Text Editions, Springer-Verlag, Berlin, 2001.

[8] Á.G. Horváth, On bisectors in Minkowski normed spaces, Acta Math. Hungar. 89 (3) (2000), 233-246.

[9] Á.G. HoRváth, Bisectors in Minkowski 3-space, Beiträge Algebra Geom. 45 (1) (2004), 225-238.

[10] R.C. JAmes, Orthogonality in normed linear spaces, Duke Math. J. 12 (1945), $291-302$.

[11] R.C. James, Orthogonality and linear functionals in normed linear spaces, Trans. Amer. Math. Soc. 61 (1947), 265-292.

[12] R. Klein, "Concrete and Abstract Voronoi Diagrams", Lecture Notes in Computer Science, Springer-Verlag, Berlin, 1989.

[13] Lihong MA, "Bisectors and Voronoi Diagrams for Convex Distance Functions", PhD thesis, Fern-Universität Hagen, 2000.

[14] H. Martini, K.J. Swanepoel, The geometry of Minkowski spaces-a survey, II, Expo. Math. 22 (2) (2004), 93-144.

[15] H. Martini, K.J. Swanepoel, G. Weiss, The geometry of Minkowski spaces-a survey, I, Expo. Math. 19 (2) (2001), 97-142.

[16] H. Martini, S. Wu, Radial projections of bisectors in Minkowski spaces, Extracta Math. 23 (1) (2008), 7-28.

[17] A. Skorik, M. ZaidenberG, On isometric reflections in Banach spaces, Mat. Fiz. Anal. Geom. 4(1-2) (1997), 212-247.

[18] A.C. Thompson, "Minkowski Geometry", Encyclopedia of Mathematics and its Applications, 63, Cambridge University Press, Cambridge, 1996. 